\title{
ОЦЕНОЧНЫЕ КОММЕНТАРИИ В НОВОСТНОМ ДИСКУРСЕ
}

\author{
О. Н. Зенищева \\ Воронежский государственный университет
}

EVALUATIVE COMMENT IN NEWS DISCOURSE

\author{
O. N. Zenishcheva \\ Voronezh State University
}

\begin{abstract}
Аннотация: статья посвящена анализу оценочных комментариев в сфере новостного дискурса. Лингвстическое изучение оченочных комментариев, входящих в состав пользовательского отклика, актуально в силу малой изученности новых жанров, специфичных для компьютерно-опосредованной коммуникации (КОК), на фоне ее интенсивного развития на современном этапе. Изучение оценочных комментариев представляет интерес не только в рамках одного языка, но и в переводческом аспекте в связи с ростом общественного интереса не только к официальным новостным публикациям, но и к реакциям широкой аудитории иноязычных пользователей на то или иное событие, особенно, если это событие произошло в стране адресатов перевода. Целью исследования является изучение особенностей оиеночного комментария в сетевом новостном дискурсе. Предметом анализа является специфика функиионирования прилагательных в составе оценочного комментария. На материале новостных публикаций на сайтах CNN и ВВС, посвященных трагическому событию, когда в средней школе города Парклэнд в американском итате Флорида произошло массовое убийство школьников и учителей, проводится речеактовый анализ, фреймовый анализ, структурный анализ, сопоставительный анализ, а также количественный анализ. Проведенное исследование позволяет сделать вывод о том, что при реализации оценочного комментария в составе отклика на новостное сообщение на официальных новостных порталах обнаружсвается лексическая специфика. Оиеночные комментарии в составе новостного сообщения реализуются посредством определенных структур, а также оценочный комментарий реализуют реакции неодобрения («Hideоus crime!»), одобрения («Such a courageous man!»), нейтрального удивления («It's unforeseеn!») и недоверия ("That's fake news»).
\end{abstract}

Ключевые слова: компьютерно-опосредованная коммуникация (КОК), оценочный комментарий, отклик, фрейм, новостная публикация.

Abstract: the paper describes the analysis of evaluative comments in the news discourse field. The linguistic study of evaluative comments, which are the part of the user response, is very important due to the little coverage of new genres specific to computer-mediated communication, against the background of its intensive development at the present stage. The evaluative comments study is of interest not only within one language framework, but also in the translation aspect due to the growing public interest not just to official news publications, but also to a wide foreign audience reactions to this or that event, especially if this event happened in the country of the translation recipient. The aim of the research is to study the features of evaluative commentary in the network news discourse. The subject of the analysis is the specifics of the adjectives functioning as part of an evaluative commentary. Speech analysis, frame analysis, structural analysis, comparative analysis, and quantitative analysis are carried out on the material of news publications on the sites of $C N N$ and $B B C$, dedicated to the tragic event of the schoolchildren and teachers massacre in Parkland, Florida, USA. The conducted research allows us to conclude that lexical specificity is revealed when implementing an evaluative comment as part of a response to a news message on official news portals. Evaluative comments in a news message are implemented through certain structures, and evaluative comments implement reactions of disapproval ("Hideous crime!"), approval ("Such a courageous man!"), neutral surprise ("It's unforeseen!") and mistrust ("That's fake news").

Key words: computer-mediated communication, evaluative commentary, response, frame, news publication.

(C) Зенищева О. Н., 2021

Контент доступен под лицензией Creative Commons Attribution 4.0 License.

The content is available under Creative Commons Attribution 4.0 License. 


\section{Введение}

В последнее время благодаря глобальной сети Интернет все более популярным становится жанр отклика. Л. Ю. Щипицина характеризует данный жанр как «читательский комментарий» и рассматривает его как часть жанра массово-информационного дискурса, а именно веб-страницы СМИ [1, с. 241]. В то же время жанр отклика характеризуется как самостоятельный жанр КОК, так как отправитель отклика отличается от отправителя сетевой публикации; при этом цель и языковые особенности данного текста являются специфичными, несмотря на расположение отклика на веб-странице СМИ [2, с. 114]. Рассмотрим жанр отклика более подробно.

\section{Результаты исследования}

Жанр отклика выделяется в работах М. А. Егоровой $[2$, с. $112 ; 3$, с. 16]. Стоит отметить вариативность обозначения термина в работах других ученых. И. Р. Танабаева использует термины «комментарий», «сетевой комментарий» или «коммент» [4, с. 207], Л. Р. Абдулина в своей работе использует термин «интернет-комментарий» [5, с. 5]. Представленные обиходные наименования мы считаем неудачными для научного обозначения исследуемого жанра, так как соответствующие тексты и их отдельные части существенно варьируются по линии коммуникативной функции, которая далеко не всегда сводится именно к комментированию поста. Таким образом, в нашем исследовании мы используем широкий термин «отклик», который никак не характеризует текст в содержательном плане, а лишь указывает на важный конститутивный признак жанра, а именно привязанность отклика к начальному посту. Отклик - это особая форма оперативного реагирования пользователей на выпуск новостей и соответствующую сетевую публикацию [3, с. 16]. Объем отклика не ограничен, но в среднем он составляет 10-20 слов (2-3 предложения).

Жанр отклика реализуется в следующих виртуальных пространствах: веб-форумы, видеохостеры, социальные сети и другие, где он традиционно называется «комментарием (к посту)». М. А. Егорова аргументирует предпочтительность использования термина «отклик» в научных целях [3, с. 16]. В работах данного автора термин «оценочный комментарий» используется для обозначения одного из речевых актов в составе отклика. Оценочный комментарий - это типичная реакция пользователей Интернета на посты, размещаемые в различных виртуальных пространствах [3, с. 112].

Перейдем к синтаксической организации откликов. Исследование было выполнено на материале новостных сообщений, посвященных трагическому событию, когда в средней школе города Парклэнд в американском штате Флорида произошло массовое убийство школьников и учителей. В рамках данного исследования нами были проанализированы 24 поста на сайтах $\mathrm{BBC}$ и $\mathrm{CNN}$ - по 12 постов на каждом, опубликованные в течение следующих 10 дней после трагедии, а также 360 пользовательских откликов на эти публикации. Объем каждого отклика не ограничен, но в среднем он составляет 10-20 слов (2-3 предложения).

Особый интерес для нас представляли оценочные комментарии в составе откликов, и в первую очередь оценочные комментарии, включающие прилагательные, общее количество которых в собранном корпусе составило 53 единицы.

Перейдем к рассмотрению базовых структур оценочного комментария, в рамках которых используются прилагательные, а также к характеристике инвентаря самих прилагательных.

На новостных сайтах $\mathrm{BBC}$ World News и CNN International нами были проанализированы структуры в основе новостных публикаций. Интерес для данного исследования представляют структуры, имеющие в своем составе оценочные слова.

В английском языке оценка события реализуется инвентарем структур, в которых в качестве оценочного слова выступают прилагательные, существительные, глаголы и наречия. Оценочное слово, входящее в состав структуры, может быть положительным (+) или отрицательным (-).

Как показывает анализ представленных данных, в основе новостных публикаций было зафиксировано 8 структур:

1) Noun + Noun in prepositional phrase: «Epidemic of mass slaughter»;

2) Noun + Adj in prepositional phrase: «The articulate anger of a bereaved parent»;

3) Adj in predicative position: «It's catastrophic»;

4) Adj + neutral Noun in attributive position: «Horrific scene», «A great guy»;

5) Adj + Noun + Noun in prepositional phrase: «Shameful display of political and civic cowardice»;

6) Adj + Noun: «Worst nightmare»;

7) Verb + Adj in prepositional phrase: «I'm devastated by this senseless shooting»;

8) Noun + Adverb: «We are deeply disturbed».

Наиболее частотной структурой и при отрицательном, и при положительном значении оценочного слова является структура «Adjective + neutral Noun in attributive position». Стоит отметить, что оценочными словами могут являться не только существительные, прилагательные и наречия, но и глагол, при котором имеет место наличие воспринимающего субъекта.

Рассмотрим типовые положительные и отрицательные структуры в основе пользовательского отклика. В результате анализа данных в основе пользо- 
вательского отклика было зафиксировано 4 структуры: «Adj in predicative position», «Adj + neutral Noun in attributive position», «Adjective + Noun», «Adverb + Adj». Наиболее частотной структурой и при отрицательном, и при положительном значении оценочного слова является структура «Adjective in predicative position».

Для подробного анализа нами было использовано 15 откликов к каждому новостному посту. Отклик может содержать широкий спектр речевых актов. Путем речеактового анализа в составе изучаемых откликов нами были выделены оценочные комментарии, а из их числа оценочные комментарии, содержащие прилагательные.

По семантике М. А. Егорова [2, с. 113] подразделяет оценочные комментарии на реакции одобрения, неодобрения, нейтрального удивления и недоверия.

Оценочный комментарий реализуется инвентарем структур, в которых в качестве оценочного слова выступают прилагательные, существительные, глаголы и наречия.

Интересным является вопрос о том, какие именно факторы влияют на характер оценочного комментария. Последний, однако, не задает характер содержания оценочного комментария в жесткой форме.

По мнению М. А. Егоровой [2, с. 116], на содержание оценочного комментария влияют как минимум 3 фактора.

1. Характер жизненной ситуации:

1) однозначно оцениваемые - положительные или отрицательные;

2) противоречивые - в центре внимания как плюсы сложившейся ситуации, так и минусы.

2. Личностный фактор:

1) возрастные различия;

2) гендерные различия;

3) статусные различия;

4) несовпадение политических взглядов;

5) несовпадение ценностных ориентиров.

3. Позиция, с которой оцениваются события.

1) общечеловеческие ценности;

2) групповая выгода;

3) личная выгода.

Главными участниками новостного события являются работники ВВС (BBC Crew), в числе которых М. А. Егорова [2, с. 16] выделяет команду сотрудников, работающих в студии, репортеров на месте событий, а также непосредственно составителей сайта BBC World Service. Они выступают в роли отправителей информации. В роли получателей информации выступают телезрители.

Главными участниками дискурсивного события отклика являются его автор (говорящий), входящий в число реципиентов новостной информации, и адресаты отклика, в числе которых выделяются следую- щие категории: любые из участников реального события, отправители новостной информации, а также другие зрители - рядовые пользователи социальной сети Facebook. При этом отклик может быть адресован как конкретному лицу в числе рядовых пользователей, так и общей массе пользователей. Интересно, что некоторые отклики могут быть адресованы всему населению планеты, т. е. иметь глобальный характер [3, с. 17].

Далее рассмотрим результаты проведенного нами анализа оценочных комментариев в новостном дискурсе.

В связи с тем, что в рамках данного исследования нами были выбраны два новостных портала (ВВС и $\mathrm{CNN}$ ), мы провели общую сравнительную характеристику новостных постов на данных новостных порталах.

Рассмотрим особенности освещения изучаемого события двумя вещательными компаниями - BВС и CNN. C этой целью нами были проанализированы 24 поста на сайтах указанных компаний - по 12 постов на каждом. Анализ показывает наличие как сходств, так и различий. Как сходства, так и отличия обнаруживаются в объеме и компонентном составе поста.

Начнем с характеристики компонентов. Современную публикацию отличает прежде всего ее мультимедийный характер: помимо текстовой составляющей, в посте широко представлены видео/аудиоматериалы и фотографии. Второе важное свойство - гипертекстовость. Современный новостной пост предполагает наличие ссылок на предыдущие публикации, гипертекстовые ссылки на другие источники, в частности, сайты упоминаемых организаций. Если рассматривать текстовый компонент, то здесь обращает на себя внимание наличие прямой речи наряду с журналистским текстом. Это новая черта современной журналистики, которая не претендует быть единственным авторитетом в изложении материала и активно привлекает мнение читательской аудитории, включая как рядовых читателей, так и важных персон. Таким образом, можно сказать, что освещение события дается одновременно с различных точек зрения при максимальной нейтральности самого журналиста. Прямая речь включает фрагменты интервью, цитаты из речей и выступлений (полная запись выступления может быть представлена в виде видеоматериала), скриншоты публикаций на личных страницах в соцсетях, в основном в Твиттере. Появление скриншотов и их достаточно широкое распространение - новая черта сетевой журналистики.

Количественный анализ показывает, что средний объем постов на ВВС и CNN составляет 5542 знака. При этом журналистский текст на ВВС составляет $76,9 \%$ от общего количества знаков, а прямая речь $23,1 \%$. На CNN - 78,9 и 21,1 \% соответственно. 
Среднее количество видео составляет 3 единицы, средняя продолжительность которых - 2,5 минуты. Количество видео, содержащих различного рода интервью, преобладает и является типичным. Это объясняется тем, что при сообщении информации от первого лица новостная компания передает ответственность за переданную информацию говорящему. Отличительной особенностью $\mathrm{CNN}$ является то, что вслед за основным, автоматически воспроизводятся 6-7 видео из других, более ранних публикаций. Попутно отметим, что из общей статистики выбиваются специализированные посты, например, содержащие только выступление или интервью. Это влияет как на объем поста, так и на процентное соотношение журналистского текста и прямой речи.

Стоит отметить, что на британском новостном портале ВВС в указанный период времени не было обнаружено постов, которые содержали бы в себе исключительно прямую речь, в то время как на американском новостном портале CNN были зарегистрированы 3 новостные публикации такого рода. Это объясняется прежде всего тем, что новостные публикации на CNN затрагивают вопросы внутренней политики, релевантной именно для США. Следовательно, фактор месторасположения вещательной компании влияет на отбор публикуемого материала.

Перейдем к сходствам и различиям в содержательном плане. Одним из основных различий между $\mathrm{BBC}$ и $\mathrm{CNN}$ является широта охвата фрейма события. Под фреймом мы понимаем модель ситуации, которая порождает зрительский отклик [6]. Анализ новостных публикаций на сайте CNN, в частности, показывает, что они имеют более широкий охват фрейма события, чем новостные публикации на сайте ВВС. Это проявляется в разном количестве непосредственных и опосредованных участников последнего, рассматриваемых в ходе освещения события. Так, в публикациях ВВС не обнаружено упоминаний или цитирований таких участников, как семья и окружение стрелявшего, жители города, полицейские, врачи в больнице, правительство штата и водитель такси. Данное различие также связано с разной степенью релевантности происшествия для жителей США и читательской аудитории за пределами страны, которым адресовано сообщение на новостном портале BBC.

Дополнительно за счет расширения фрейма поддерживается интерес публики к данному новостному событию, поскольку это позволяет сохранять новизну сообщаемой информации. Данное количественное превосходство групп участников непосредственно оказывает влияние на количество «лайков» и откликов на каждом новостном портале. В результате количественного анализа нами было выявлено, что среднее количество «лайков» на новостном портале ВВС составляет 3040, в то время как на $\mathrm{CNN}$ - 23572. Стоит также отметить, что среднее количество откликов на ВBC и CNN составляет 533 и 1389 откликов соответственно.

Такое количественное различие может быть также обусловлено постепенным обновлением деталей случившегося и последующих предпринятых действий. Стоит отметить, что стимулом для отклика является новостное сообщение, за которым стоит реальная ситуация.

Исходной точкой фрейма в каждом случае является само реальное событие, которое, прежде всего, предполагает участников. Последние, как показывает анализ, в зависимости от степени их участия подразделяются на непосредственных и опосредованных, т. е. либо не присутствовавших на месте реального события, либо непосредственно не затронутых данным событием. Состав и количество участников варьируется. Реальное событие конкретно локализовано в пространстве и во времени.

Перейдем к общей сравнительной характеристике откликов на новостные сообщения на порталах $\mathrm{BBC}$ и $\mathrm{CNN}$.

Новостной пост на сайте новостного портала и в социальных сетях является новым жанром, специфичным для компьютерно-опосредованной коммуникации. Его отправителем является вещательная компания. Социальные сети предоставляют техническую возможность комментирования новостных публикаций пользователями социальных сетей. Текст, расположенный под постом, отправителем которого является пользователь, мы называем «откликом». На наш взгляд, отклик следует характеризовать как самостоятельный жанр компьютерно-опосредованной коммуникации, так как отправитель отклика отличается от отправителя новостной публикации; специфичны при этом и цель, и языковые особенности данного текста, несмотря на его расположение на веб-странице СМИ. Еще одним аргументом в пользу выделения отклика в самостоятельный жанр является тот факт, что нередко отклик - это реакция не на сетевую публикацию, а на соответствующий видеоматериал. На рассмотренных нами новостных порталах $\mathrm{BBC}$ и $\mathrm{CNN}$ пользовательские отклики периодически цитируются в ходе новостных программ, что также свидетельствует как об их относительной самостоятельности, так и об отсутствии жесткой привязки к самой сетевой публикации - отклик, прежде всего, является реакцией на определенное событие, на позицию канала в отношении этого события, и уже в дополнение к этому - на конкретную форму сообщения об этом событии (в сети / по телевидению). В рамках данного исследования нами было отобрано 360 пользовательских откликов на новостных порталах $\mathrm{BBC}$ и CNN. Анализ показывает наличие как 
сходств, так и различий в объеме и компонентном составе отклика.

Начнем с характеристики компонентов. Отклик пользователя отличает низкая степень гипертекстуальности, так как в тексте отклика пользователи редко добавляют гиперссылки. Наличие разнообразных смайлов указывает на мультимедийность. В связи с тем, что на каждый пользовательский отклик отправитель отклика может получить соответствующую реакцию (данная черта характеризуется наличием под каждым откликом кнопки «Like»), можно сделать вывод о высокой степени потенциальной интерактивности. Еще одной характерной чертой является дистантность коммуникантов, которая обусловливается возможностью взаимодействия посредством компьютера, при котором возникает возможность общения людей, лично не знакомых друг с другом. Стоит также отметить эксплицированный тип коммуникантов, поскольку пользователю необходимо пройти процесс регистрации на веб-сайте, прежде чем ставить лайки или комментировать новостной пост.

В результате количественного анализа нами было выявлено, что средний объем отклика на ВВС составляет 15 слов (1-2 предложения), в то время как на $\mathrm{CNN}$ средний объем отклика составляет 30-40 слов (4-5 предложений). Данное различие связано с разной степенью релевантности происшествия для жителей США и читательской аудитории за пределами страны, которым адресовано сообщение на новостном портале ВВС.

В результате анализа пользовательских откликов нами было выявлено, что посредством речевого акта, называемого оценочным комментарием, пользователи дают оценку события. Событие, рассмотренное нами (массовое убийство школьников), вызвало большой общественный резонанс и широкий спектр реакций, включая оценочный комментарий. Приведем несколько ярких примеров последних:

What a tragedy! / What a horrible crime! / So sad! / Bad news! / Too bad!

Оценочный комментарий неразрывно связан с новостным постом, который не детерминирует оценочный комментарий в жесткой форме, а лишь в определенной степени влияет на его содержание, оставляя значительную свободу выбора автору комментария. В силу действия личностного фактора (возрастные, гендерные, статусные различия, несовпадение политических взглядов и ценностных ориентиров) оценочные комментарии к одному и тому же событию у разных коммуникантов зачастую существенно различаются. Расхождение точек зрения может также являться следствием оценки события с различных позиций: с позиции общечеловеческих ценностей и с позиции групповой или личной выгоды, далеко не всегда совпадающих друг с другом. Нередко причиной несовпадения оценок является противоречивый характер самих жизненных ситуаций, например, как в нашем случае попытка достижения благих целей путем предложения альтернативного решения проблемы. Последнее, в частности, имело место при оглашении президентом Дональдом Трампом предложения о вооружении учителей в американских школах. Данное событие вызвало широкий спектр реакций: от «Good!» и «Better than nothing» до «It's madness!» и «Stupid idea!».

Неоднозначные события, как правило, предполагают наличие некоторого пострадавшего меньшинства. Обращает на себя внимание, что в таких случаях заметно возрастает частотность структуры $A d j+$ Noun! Эта тенденция, к примеру, четко прослеживается в комментариях по поводу посмертного вручения медалей погибшим школьникам и учителям, где прилагательные great, amazing, awesome в основном употребляются не изолированно, а в составе именно этой структуры, что позволяет перенести акцент на положительное событие, избежав оценки основного события (массового убийства школьников).

Помимо характера события, определяемого в терминах интересов и шкал ценностей (good-bad), на содержание и языковое оформление комментария влияют такие признаки, как масштаб события (catastrophic, terrifying - sad, too bad) и степень его непредсказуемости (Surprise! - Finally!). Единицы Finally! и At last! используются для выражения одобрения событий или раздражения, которому предшествовало долгое ожидание. Их целесообразно отнести к косвенным языковым средствам реализации оценочного комментария, поскольку оценочный компонент не входит в состав их лексической семантики.

Как показывает анализ корпуса, оценочные комментарии используются в двух социальных функциях: для обозначения личной позиции автора оценочного комментария по отношению к событию и для выражения его сопричастности происходящему.

Первая функция реализуется в комментариях событий, характеризующихся неоднозначностью, которая, как было показано выше, проистекает из наличия у события как позитивной, так и негативной сторон. В таких случаях оценочные комментарии разных пользователей могут оказаться прямо противоположными. Так, если для определенной группы пользователей факт наличия пострадавшего меньшинства перевешивает по значимости факт наличия благоприятного исхода для большинства, событию в целом может даваться не только положительная, но и отрицательная оценка.

События, оцениваемые с общечеловеческих позиций однозначно, достаточно жестко задают спектр 
социально-приемлемых оценочных комментариев: события, оцениваемые с общечеловеческих позиций однозначно хорошо (спасение учителями детей), предполагают реакцию одобрения, тогда как события, оцениваемые однозначно плохо (массовое убийство школьников), - реакцию неодобрения.

Обозначение личной (гражданской) позиции и выражение сопричастности следует признать основными социальными функциями оценочного комментария, полностью отвечающими прямому назначению данного виртуального пространства - служить платформой для серьезного, открытого обсуждения мировых событий. Сказанное, однако, не означает, что у отдельных пользователей может не быть иных, в том числе и идущих вразрез с целью создания подобных платформ, мотивов.

По семантике оценочные комментарии, как указывалось выше, подразделяются на реакции одобрения, неодобрения, нейтрального удивления и недоверия. Наличие последней объясняется тем, что оценочный комментарий может соотноситься как с самим событием, так и с сообщением об этом событии на телеканале.

Общее количество разных прилагательных в корпусе составило 53 лексических единицы. Также было зафиксировано 477 употреблений (включая случаи повторения) данных прилагательных в рассмотренных нами новостных сообщениях и пользовательских откликах. Наибольшее разнообразие инвентаря прилагательных зарегистрировано в случае реакции неодобрения (37 единиц, 359 употреблений).

В их числе можно выделить как общеоценочные прилагательные, например: «That's bad!», «So sad...», так и частнооценочные прилагательные, например: «It's a fearfull moment!», «Such a frightful news!».

На втором месте с точки зрения разнообразия используемых единиц оказались прилагательные, реализующие реакцию одобрения (6 единиц, 38 употреблений) и нейтрального удивления (6 единиц, 26 употреблений): «Such a courageous man», «This is surreal». Существенное отличие количества единиц, реализующих реакцию неодобрения по сравнению с реакциями одобрения и нейтрального удивления, объясняется характером события (массовое убийство в средней школе). Реакции одобрения были зарегистрированы только в пользовательских откликах к новостным публикациям, где была представлена информация о героических поступках погибших.

В первом из двух примеров использовано частнооценочное прилагательное courageous. Другие частнооценочные прилагательные включают такие единицы, как «Brave man!», «Amazing people!». Во втором примере использовано общеоценочное прилагательное surreal.
Значительно меньшее число единиц в корпусе реализуют реакции недоверия. В данном случае это 3 прилагательных (24 употребления), например: «It's all fake news!».

Проведенный анализ показывает, что оценочный комментарий типично реализуется частнооценочными прилагательными.

Наряду с единицами, специализирующимися на выражении определенного рода реакции, в корпусе обнаруживаются единицы с весьма широким диапазоном употребления: прилагательные amazing и unbelievable. Первое способно выражать спектр значений от одобрения и одобрения в сочетании с удивлением до удивления с оттенком любопытства и удивления с оттенком осуждения. Второе может выражать недоверие и неодобрение с оттенком удивления; при этом оно также используется для выражения удивления с оттенком радости, наряду с прилагательным incredible. Для интерпретации этих двух единиц необходимо привлечение контекста, в том числе и ситуативного.

В ходе исследования нами также решалась задача количественного анализа семантических вариантов оценочного комментария.

В ходе анализа было выявлено, что реакции неодобрения являются наиболее частотным видом оценочного комментария (37 случаев), на втором месте по частотности оказались реакции одобрения (6 случаев) и реакции нейтрального удивления (6 случаев), значительно реже в корпусе встречаются реакции недоверия (3 случая).

Рассмотрим оценочные комментарии из собранного корпуса с точки зрения их референциальной отнесенности.

В результате изучения отнесенности оценочного комментария были выявлены следующие случаи.

1. Оценочные комментарии, относящиеся к реальному событию в целом:

«That is so terrible!»

«So awful!»

"It is horrible!! And this has happened at school!»

2. Оценочные комментарии, относящиеся к отдельным участникам реального события:

"They seem really aggressive.»

«Spiteful people!!»

«He is a lunatic!!»

3. Оценочные комментарии, относящиеся к месту действия события:

"America is a scary place to live in.»

"This school is a terrifying place!»

4. Оценочные комментарии, относящиеся к говорящему и другим участникам событий:

«These kids are so brave!» 
5. Оценочные комментарии, относящиеся к новостному событию в целом:

«That's really insane!»

6. Оценочные комментарии, относящиеся к отдельным участникам новостного события:

"CNN has an incredible way of presenting the news.»

7. Оценочные комментарии, относящиеся к фотографиям в составе новостного сообщения:

\section{«Catastrophic!» \\ «Dreadful!».}

Одной из задач исследования является количественный анализ оценочного комментария с точки зрения их референциальной отнесенности.

В ходе анализа было выявлено, что чаще всего оценочный комментарий соотносится с реальным событием (71 случай). На втором месте по частотности оценочные комментарии, относящиеся к участникам события (28 случаев). В 10 случаях оценочные комментарии соотносятся с фотографиями, а не с самим событием. Это имело место в случае комментариев, относящихся к новостным публикациям, которые содержали фотографии погибших школьников и учителей.

Значительно меньшее количество оценочных комментариев имеют отнесенность к новостному событию (9 случаев), к месту (2 случая) и к говорящему (2 случая).

Также нами было изучено использование прилагательных в составе новостного сообщения и пользовательского отклика. Ниже представлены прилагательные, реализующие оценочный комментарий в составе новостного сообщения и пользовательского отклика в зависимости от его референциальной отнесенности.

Прилагательные, реализующие оценочный комментарий, имеющий отнесенность к новостному событию: heartbreaking, unspeakable, unbelievable, shocking, shameful.

Прилагательные, реализующие оценочный комментарий с отнесенностью к реальному событию: shocking, catastrophic, heartbreaking, dreadful, terrible, sudden, fearful, horrible, tragic, frightful, awful, terrifying, painful, sad, scary, insane, unbelievable, outrageous, disgusting.

Оценочный комментарий, имеющий отнесенность к участникам события: aggressive, alarming, sick, spiteful, innocent, disgusting, lunatic, crazy, mad.

Прилагательные, реализующие оценочный комментарий, имеющий отнесенность к месту: scary, terrible.

Прилагательные, реализующие оценочный комментарий с отнесенностью к фото: unbelievable, shocking, scary, mind-boggling.

Оценочный комментарий, имеющий отнесенность к говорящему: speechless, selfish, incompetent.

\section{Выводы}

Анализ корпуса прилагательных позволяет сделать следующие выводы.

1. Набор используемых прилагательных существенно варьируется в зависимости от референциальной отнесенности оценочного комментария. Например, такое прилагательное, как outrageous, используется для реализации реакции неодобрения, в аналогичной функции используются прилагательные shocking и catastrophic. Это связано с тем, что прилагательное outrageous описывает целенаправленные действия, в то время как прилагательные shocking и catastrophic описывают неожиданные события, не зависящие от действий людей и происходящие помимо их воли, подчеркивая масштаб трагедии.

Когда действия имеют осознанный и целенаправленный характер (например, решение президента Дональда Трампа вооружить учителей), нами было зафиксировано использование таких единиц, как disgusting, outrageous, характеризующих непорядочное поведение участников.

2. Выбор прилагательных зависит от референциальной отнесенности оценочного комментария (speechless - соотнесенность с говорящим, unspeakable - соотнесенность с событием). Реакции неодобрения, относящиеся к участникам, реализуются такими прилагательными, как aggressive, incompetent, selfish, характеризующими отрицательное поведение людей. В то же время реакции неодобрения, относящиеся к реальному событию, реализуются прилагательными horrible, awful, dreadful. Прилагательное speechless соотносится в данной области с говорящим, в то время как unspeakable характеризует новостное событие.

3. У ряда прилагательных, и прежде всего у прилагательного unbelievable, значение в определенной степени модифицируется в зависимости от контекста. Например, в составе отклика «Unbelievable! It just can not be true!», прилагательное unbelievable реализует реакцию нейтрального удивления, приобретая при этом положительное значение. В то время как в составе отклика «This is just unbelievable! How could this happen to the children?! "), данное прилагательное реализует реакцию неодобрения, приобретая тем самым отрицательное значение.

\section{ЛИТЕРАТУРА}

1. Щипицина Л. Ю. Компьютерно-опосредованная коммуникация : Лингвистический аспект анализа : монография. М. : КРАСАНД, 2010. 296 с.

2. Егорова М. А. Оценочный комментарий в социальных сетях (на материале комментариев к новостным постам) // Современная филология : теория и практика. М. : Институт стратегических исследований, 2013. C. $112-118$. 
3. Егорова М. А. Отклики и оценочные комментарии при КОК // Вестник Воронеж. гос. ун-та. Сер.: Лингвистика и межкультурная коммуникация. 2014. № 1. C. 16-20.

4. Танабаева И. Р., Нестерик Э. В. Комментарий как особый жанр в интернет-общении // Молодой ученый. Сер.: Филология. 2017. № 51. С. 207-209.

5. Абдуллина Л. Р. Интернет-комментарий как отражение национального мировосприятия // Вестник Воронеж. гос. ун-та. Сер.: Филология. Журналистика. 2016. № 1. C. 5-6.

6. Стренева Н. В. Понятийный потенциал термина «фрейм». URL: http://vestnik.osu.ru/2009_11/13.pdf

\section{ИСТОЧНИКИ}

1. BВC World News в социальной сети Facebook. URL: http://www.facebook.com/bbcnews

2. CNN International в социальной сети Facebook. URL: https://www.facebook.com/cnninternational

3. Агентство международной информации ВВС World News. URL: http://www.bbc.co.uk

4. Агентство международной информации CNN International. URL: https://edition.cnn.com

5. НКРЯ - Национальный корпус русского языка. URL: http://www.ruscorpora.ru

\section{REFERENCES}

1. Shchipitsina L. J. Komp'yuterno-oposredovannaya kommunikatsiya: Lingvisticheskij aspekt analiza [Computer-mediated communication: Linguistic aspect of analysis]: monograph. M.: KRASAND, 2010. 296 p.

2. Egorova M. A. Otsenochnyj kommentarij v sotsial'nykh setyakh (na materiale kommentariev k novostnym

Воронежский государственный университет

Зенищева О. Н., соискатель кафедры теоретической и прикладной лингвистики

E-mail: olesyazenishcheva@gmail.com

Поступила в редакиию 1 февраля 2021 г.

Принята к публикации 22 марта 20212.

\section{Для цитирования:}

Зенищева О. Н. Оценочные комментарии в новостном дискурсе // Вестник Воронежского государственного университета. Серия: Лингвистика и межкультурная коммуникация. 2021. № 2. C. 15-22. DOI: https://doi. org/10.17308/lic.2021.2/3411 postam) [Evaluative comment in social networks (based on the news posts comments)]. In: Sovremennaya filologiya: teoriya i praktika. M.: Institut strategicheskikh issledovanij, 2013. Pp. 112-118.

3. Egorova M. A. Otkliki i otsenochnye kommentarii pri KOK [Responses and evaluative comments in computer-mediated communication]. In: Vestn. Voronezh. gos. un-ta. Ser.: Lingvistika i mezhkul'turnaya kommunikatsiya. 2014. No. 1. Pp. 16-20.

4. Tanabaeva I. R., Nesterik EH. V. Kommentarij kak osobyj zhanr v internet-obshhenii [Commentary as a special genre in Internet communication]. In: Molodoj uchenyj. Ser.: Filologiya. 2017. No. 51. Pp. 207-209.

5. Abdullina L. R. Internet-kommentarij kak otrazhenie natsional'nogo mirovospriyatiya [Internet commentary as a reflection of the national perception of the world]. In: Vestn. Voronezh. gos. un-ta. Ser.: Filologiya. ZHurnalistika. 2016. No. 1. Pp. 5-6.

6. Streneva N. V. Ponyatijnyj potentsial termina «frejm» [Conceptual potential of the term "frame"]. Available at: http://vestnik.osu.ru/2009_11/13.pdf

\section{SOURCES}

1. BBC World News on Facebook. Available at: http:// www.facebook.com/bbcnews

2. CNN International on Facebook. Available at: https:// www.facebook.com/cnninternational

3. BBC World News. Available at: http://www.bbc. co.uk

4. CNN International. Available at: https://edition.cnn. com

5. RNC - Russian National Corpus. Available at: http:// www.ruscorpora.ru

Voronezh State University

Zenishcheva O. N., Post-graduate Student, of the Theoretical and Applied Linguistics Department

E-mail: olesyazenishcheva@gmail.com

Received: 1 February 2021

Accepted: 22 March 2021

\section{For citation:}

Zenishcheva $O$. N. Evaluative comment in news discourse. Proceedings of Voronezh State University. Series: Linguistics and Intercultural Communication. 2021. No. 2. Pp. 15-22. DOI: https://doi.org/10.17308/lic.2021.2/3411 